\title{
Effects of amlodipine on bone metabolism in male albino Wistar rats
}

\author{
Iveta Gradošová ${ }^{1}$, Helena Živná ${ }^{2}$, Klára Švejkovská ${ }^{1}$, Vladimír Palička ${ }^{1}$, Aleš Tichý ${ }^{3}$, \\ Pavel Živný ${ }^{1}$
}
${ }^{1}$ Institute of Clinical Biochemistry and Diagnostics, Medical Faculty and University Hospital, Hradec Králové, Czech Republic
${ }^{2}$ Radioisotope Laboratories and Vivarium, Faculty of Medicine in Hradec Králové, Charles University in Prague, Czech Republic
${ }^{3}$ Department of Radiobiology, University of Defence, Faculty of Military Health Sciences, Hradec Králové, Czech Republic

Received May 6, 2011

Accepted December 14, 2011

\begin{abstract}
Amlodipine (dihydropyridine-type calcium channel blocker) is a widely used agent for the treatment of hypertension in human and veterinary medicine but detailed information about its effects on bone metabolism are missing. Therefore, the aim of our study was to investigate the effect of amlodipine on bone metabolism in male albino Wistar rats. Amlodipine $(0.3 \mathrm{mg} / 100 \mathrm{~g}$ body weight; gavage) was administered to 8 rats for 8 weeks. Control group $(n=8)$ received aqua pro inj. ( $0.2 \mathrm{ml} / 100 \mathrm{~g}$ body weight; gavage). Bone marker concentrations of carboxy-terminal cross-linking telopeptide of type I collagen (CTX-I) and aminoterminal propeptide of procollagen type I in serum, and of bone alkaline phosphatase (BALP) in both serum and bone homogenate were measured by enzyme immunoassay. We investigated the expression of bone morphogenetic protein 2 (BMP-2) in proximal tibia using Western blotting, and bone mineral density was measured by Dual-energy X-ray Absorptiometry in lumbar and caudal vertebrae and in femoral areas. Mechanical properties of the femurs were measured by three-point bending of the shaft and compression testing of the femoral neck. After 8 weeks of amlodipine administration there was a significant decrease in serum concentrations of BALP $(p=0.0009)$ and CTX-I $(p=0.003)$, and the content of BALP in bone homogenate $(p=0.026)$ compared to the control. In addition, Western blot analysis indicated increased BMP-2 protein concentration after amlodipine administration. Our findings suggest that amlodipine has a retarding influence on bone metabolism in rats by decreasing bone turnover, which probably in consequence increases expression of BMP-2.
\end{abstract}

Bone markers, Western blotting, bone mineral density, biomechanical testing

Cardiovascular diseases are one of the leading causes of death for people in developed countries world-wide. The most common form is arterial hypertension, which can be reduced by several groups of drugs. At the present, not much attention has been paid to whether or how these drugs affect bone metabolism.

Hypertension and osteoporosis are frequent diseases among people of higher age. Both are induced by interaction of many genetic and environmental factors (Shimizu et al. 2008). The first-choice antihypertensive drugs include the calcium channel blockers. Amlodipine is a derivate of dihydropyridine and is one of the $3^{\text {rd }}$ generation calcium channel blockers. Amlodipine is effectively used in human and veterinary medicine. Its principal action is to inhibit calcium entry through voltage-gated transmembrane L-type channels, thus decreasing intracellular calcium concentration and inducing smooth muscle relaxation (Devabhaktuni et al. 2009).

Osteoblasts are derived from mesenchymal stem cells and play a pivotal role in bone formation. Osteoblasts have been also found to exprimate voltage-gated calcium channels (Duncan et al. 1998). L-type calcium channels have been shown in a rat osteoblast-like cell line (Morain et al. 1992). Vitamin $\mathrm{D}_{3}$, parathyroid hormone

Address for correspondence:

Mgr. Iveta Gradošová

Institute of Clinical Biochemistry and Diagnostics

Medical Faculty and University Hospital

Sokolská 581, 50002 Hradec Králové, Czech Republic
Phone: +420495816337

Fax: + 420495832003

E-mail: gradosovai@lfhk.cuni.cz

http://www.vfu.cz/acta-vet/actavet.htm 
and prostaglandin $\mathrm{E}_{2}$ regulate bone metabolism. They also cause an increase in the intracellular calcium concentration, at least part of which is decreased by the action of dihydropyridines (Lieberherr 1987). These regulators of bone metabolism also promote or inhibit osteoblast differentiation (Nakatani et al. 1984; Kurihara et al. 1986). Regulators of bone formation modulate also the expression of L-type calcium channels and their functional involvement in bone remodeling ( $\mathrm{Gu}$ et al. 2001). During the differentiation of bone mesenchymal stem cells of human bone marrow, the voltage-gated calcium channels could be a target for pharmacological regulation of this process (Zahanich et al. 2005). This fact indicates an important role for calcium channels in the regulation of osteoblast function and bone metabolism.

Many studies indicate that calcium channel blockers influence bone metabolism, but at present there is no agreed opinion about whether they are beneficial or not. Most studies have demonstrated a somewhat positive effect on bone tissue resulting in a decrease in bone resorption, an increase in bone mineral density (BMD) and a reduction of risk for fractures (Ritchie et al. 1994; Rejnmark et al. 2006; Ushijima et al. 2010). So far, little information has been published on the effect of amlodipine on bone metabolism (Nishiya and Sugimoto 2001; Halici et al. 2008; Ushijima et al. 2010).

The present study aimed to determine the effect of amlodipine $(0.3 \mathrm{mg} / 100 \mathrm{~g}$ body weight $\mathrm{BW}$ ) on bone metabolism in male albino Wistar rats by examining the markers of bone turnover: carboxy-terminal cross-linking telopeptide of type I collagen (CTX-I), bone alkaline phosphatase (BALP), and aminoterminal propeptide of procollagen type I (PINP). We also examined the expression of bone morphogenetic protein 2 (BMP-2) using the Western blot method, measured the bone mineral density (BMD), and tested mechanical properties of the femurs.

\section{Materials and Methods}

\section{Animals}

The experimental protocol was approved by the Animal Welfare Committee of Charles University in Prague, Faculty of Medicine in Hradec Králové (No. 2709/2009-30). Animals were housed in the Vivarium of the Faculty of Medicine in Hradec Králové. In the experiment, we used 16 male albino Wistar rats (Biotest s.r.o., Konárovice, Czech Republic) weighing $240 \pm 10 \mathrm{~g}$, two months old at the beginning of the experiment; they were divided into two groups of 8 animals. Rats were placed in groups of 4 in plastic cages and kept under standard conditions. They received the standard laboratory diet (ST-1, VELAS, a.s., Lysá nad Labem, Czech Republic) and drinking water ad libitum.

\section{Experimental design}

The control group was given aqua pro injectione (Aqua, Fresenius Kabi Italia S. r. 1., Verona, Italy) at a dose of $0.2 \mathrm{ml} / 100 \mathrm{~g}$ body weight of rat. The experimental group received amlodipine (Cardilopin, Egis Pharmaceuticals PLC, Budapest, Hungary) at a dose of $0.3 \mathrm{mg}$ in $0.2 \mathrm{ml}$ aqua pro inj./100 g body weight. The amlodipine and aqua pro inj. were applied orally by gavage daily in the morning for 8 weeks. The animals were weighed every day to adjust the amlodipine dose to body weight.

Bone mineral density measurements

After an 8-week administration of amlodipine/aqua pro inj., the rats were placed under analgosedation $(5 \mathrm{mg} / \mathrm{kg}$ of midazolam i.m. Midazolam Torrex 5mg/ml; Torrex Chiesi Pharma GMBH, Vienna, Austria and $100 \mathrm{mg} / \mathrm{kg}$ ketamine i.m. Narkamon Spofa 1\%; SPOFA a.s. Prague, Czech Republic) and their bone mineral density (BMD, g/ $\mathrm{cm}^{2}$ ) was measured by means of Dual-energy X-ray Absorptiometry (DXA) on a Hologic Delphi A device at the Osteocentre of the Faculty Hospital Hradec Králové. Before measurements, a tissue calibration scan was performed with the Hologic phantom for the small animal. Bone mineral density was evaluated in lumbar and caudal vertebrae and in femoral areas by an appropriate PC program (DXA; QDR-4500A Elite; Hologic, Waltham, MA, USA).

\section{ELISA}

The animals $(460 \pm 30 \mathrm{~g} \mathrm{BW})$ were sacrificed under ether anaesthesia by withdrawal of blood from the bifurcation of the abdominal aorta. The obtained blood serum served to determine the concentrations of the markers of bone turnover: CTX-I, PINP and the BALP activity. 
Both tibias were carefully excised from the animals and bone segments of the proximal region were obtained. Frozen samples $(100 \mathrm{mg})$ were minced in a phosphate buffer $(1.5 \mathrm{ml})$ and then disrupted and homogenized in a MagNA Lyser instrument (Roche Applied Science, Germany) during three rounds at $6500 \mathrm{rpm}$ for $20 \mathrm{~s}$ with intermediate cooling $5 \mathrm{~min}$ in the MagNA Lyser Cooling Block.

Serum concentrations of bone markers CTX-I and PINP, and the BALP concentration in serum and bone homogenate were assayed using a commercial rat Enzyme-Linked Immunosorbent Assay (ELISA) kit manufactured by the firm Immunodiagnostic Systems Ltd., Great Britain (CTX-I, RatLaps ${ }^{\mathrm{TM}}$ EIA, $\mu \mathrm{g} / 1$; PINP, Rat/Mouse PINP EIA, $\mu \mathrm{g} / \mathrm{l}$ ) and Uscnlife Sciences \& Technology Co., Ltd., China (BALP, Rat bone alkaline phosphatase, kat/l).

Biomechanical testing procedure

Biomechanical testing of the rat femurs was done with a special electromechanical custom-made testing machine (Martin Košek \& Pavel Trnečka, Hradec Králové, Czech Republic) according to methods described in our previous report (Gradosova et al. 2011).

Protein extraction, SDS-PAGE, and Western blotting

Bone homogenate was prepared from the proximal tibia for the determination of BMP-2 protein concentration by using Western blotting analysis. The samples preparation, SDS-PAGE and Western blotting were described in our previous report (Gradosova et al. 2011).

Statistical analysis

Statistics were calculated with NCSS 2007 (Number Cruncher Statistical System, Kaysville, Utah, USA). Differences between the control and experimental group were evaluated by unpaired $t$-test or Mann-Whitney test as appropriate. All experimental data were expressed as median and $25^{\text {th }}$ and $75^{\text {th }}$ percentiles. The value $p<0.05$ was considered as significant.

\section{Results}

After 8 weeks, the serum concentration of the marker of osteoresorption CTX-I in the group of rats given amlodipine showed a significant decrease to $75 \%(p=0.003)$ compared to that of the control group (Fig. 1b). The serum concentration of the marker of osteoformation PINP also showed a decrease to $73 \%(p=0.06)$ compared to that of control group (Fig. 1a). The serum concentration of BALP was enormously decreased to $12 \%(p=0.0009)$ in comparison with that of the control group (Fig. 1c). The homogenate concentration of BALP was significantly decreased to $59 \%(p=0.026)$ relative to that of control group (Fig. 1d).

Neither the tested mechanical properties of the bone tissue nor the BMD showed any significant changes compared to those of the control group after 8 weeks of amlodipine administration (Table 1). However, there was a marked increase in the concentration of protein BMP-2 in the proximal part of the tibia compared to that of the control group (Fig. 2).

Table 1. Effect of amlodipine on bone mineral density in three skeletal areas and biomechanical properties of the femur which were determined using three-point bending test and compression test of femoral neck in male albino Wistar rats

\begin{tabular}{lll}
\hline Measurement (unit) & Control group & Experimental group \\
\hline BMD lumbar vertebrae $\left(\mathrm{g} / \mathrm{cm}^{2}\right)$ & $0.217(0.213-0.224)$ & $0.218(0.214-0.220)$ \\
BMD caudal vertebrae $\left(\mathrm{g} / \mathrm{cm}^{2}\right)$ & $0.218(0.213-0.232)$ & $0.231(0.225-0.236)$ \\
BMD femoral areas $\left(\mathrm{g} / \mathrm{cm}^{2}\right)$ & $0.183(0.171-0.210)$ & $0.193(0.180-0.209)$ \\
Left femurs & & $174.00(165.00-195.00)$ \\
Maximal load of the femoral shaft $(\mathrm{N})$ & $173.50(168.25-191.00)$ & $143.00(126.50-184.00)$ \\
Maximal load of femoral neck $(\mathrm{N})$ & $144.50(118.75-161.25)$ & $185.00(182.25-194.50)$ \\
Right femurs & & $138.50(112.25-167.25)$ \\
Maximal load of the femoral shaft $(\mathrm{N})$ & $192.00(173.25-205.00)$ & $144.50(96.00-153.75)$
\end{tabular}

Values are presented as median $\left(25^{\text {th }}-75^{\text {th }}\right.$ percentiles $)$, BMD - bone mineral density, N-Newton 

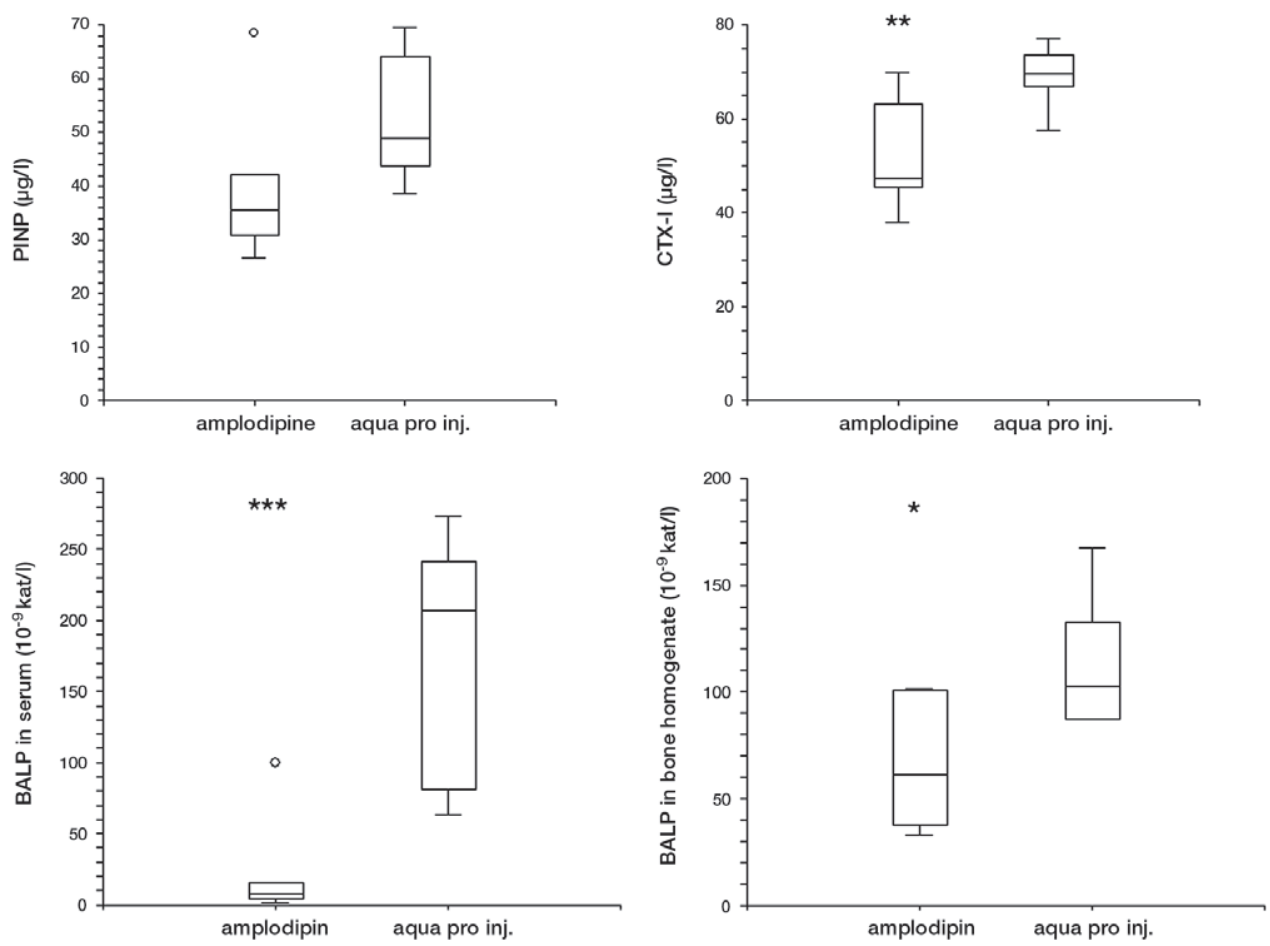

Fig. 1. Effect of amlodipine on bone formation in male albino Wistar rats after 8-week administration. The figure shows the change in amlodipine concentration compared to the control group (aqua pro inj.) of: a) aminoterminal propeptide of procollagen type I (PINP); b) carboxy-terminal cross-linking telopeptide of type I collagen (CTX-I); c) bone alkaline phosphatase (BALP) in serum; d) BALP in bone homogenate. Data are expressed as box plots. The box extends from the $25^{\text {th }}$ percentile to the $75^{\text {th }}$ percentile, with a horizontal line at the median $\left(50^{\text {th }}\right.$ percentile $)$. Whiskers extend down to the smallest value and up to the largest. Asterisks denote significant differences $(* p<0.05, * * p<0.01, * * * p<0.001)$.

\section{Discussion}

CO AML The effect of amlodipine on bone metabolism has not yet been sufficiently investigated; so far only a few studies have examined this problem (Nishiya and Sugimoto 2001; Halici et al. 2008; Ushijima et al. 2010).

After 8 weeks of amlodipine administration $(0.3 \mathrm{mg} / 100$ $\mathrm{g}$ body weight) to the rats, a significant decrease was observed in the serum concentration of CTX-I which is released from type I collagen by the action of proteolytic enzymes during its degradation into the systemic circulation (Herrmann and Seibel 2008). Ushijima et al. (2010) demonstrated a significant decrease in the serum concentration of CTX-I, in stroke-prone spontaneously hypertensive rats after a 3-month administration of amlodipine at a dose of $0.3 \mathrm{mg} / 100 \mathrm{~g}$ body weight. Dihydropyridines have been found to suppress bone resorption by direct inhibition of osteoclast activity (Ritchie et al. 1994). 
The BALP activity was surprisingly significantly decreased to $12 \%$ compared to that of the control group. The decrease of BALP in serum was verified by determination of the BALP concentration in proximal tibia homogenate using ELISA. Localization of BALP is in the membranes of the osteoblasts from which it is released into the serum during their activation (Brown et al. 2009). Aminoterminal propeptide of procollagen type I was also decreased but not significantly. The results of the present study indicate that the action of amlodipine induces suppression of bone turnover, in particular by decreasing osteoformation, and to a lesser extent osteoresorption.

After 8 weeks of amlodipine administration, an increase in expression of BMP-2 was observed in the proximal tibia. BMP-2 is the growth factor which makes possible the proliferation and differentiation of osteoblasts and the formation of bone (Katagiri and Takahashi 2002). We think that the increased concentration of protein BMP-2 is most probably a reaction to the suppression of osteoblast function by amlodipine. However, this assumption requires further studies.

Evaluation of BMD and the mechanical properties of the bone tissue have not revealed significant changes. Therefore we assume that amlodipine administration does not significantly influence bone tissue. The present results, nevertheless, indicate that amlodipine treatment in the administered dose may induce deceleration of bone metabolism.

In conclusion, long-term administration of amlodipine at a dose of $0.3 \mathrm{mg} / 100 \mathrm{~g}$ body weight exerts an effect on bone metabolism in male albino Wistar rats. The study was performed on outbred rats. In the future it will be necessary to verify the present results on rats of both sexes suffering from hypertension and induced osteoporosis.

\section{Acknowledgements}

This study was supported principally by the Research Project of the Czech Ministry of Health (MZO 00179906), by a grant from the Medical Faculty of Charles University, Hradec Králové, Czech Republic and also by the grant SVV-2010-62051.

\section{References}

Brown JP, Albert C, Nassar BA, Adachi JD, Cole D, Davison KS, Dooley KC, Don-Wauchope A, Douville P, Hanley DA, Jamal SA, Josse R, Kaiser S, Krahn J, Krause R, Kremer R, Lepage R, Letendre E, Morin S, Ooi DS, Papaioaonnou A, Ste-Marie LG 2009: Bone turnover markers in the management of postmenopausal osteoporosis. Clin Biochem 42: 929-942

Devabhaktuni M, Bangalore S: Fixed combination of amlodipine and atorvastatin in cardiovascular risk management 2009: patient perspectives. Vasc Health Risk Manag 5: 377-387

Duncan RL, Akanbi KA, Farach-Carson MC 1998: Calcium signals and calcium channels in osteoblastic cells. Semin Nephrol 18: 178-190

Gradosova I, Zivna A, Svejkovska K, Palicka V, Tichy A, Zivny P 2011: The role of atorvastatin in bone metabolism in male albino Wistar rats. Pharmazie 66: 606-610

Gu Y, Preston MR, Magnay J, El Haj AJ, Publicover SJ 2001: Hormonally-regulated expression of voltageoperated $\mathrm{Ca}(2+)$ channels in osteocytic (MLO-Y4) cells. Biochem Biophys Res Commun 282: 536-542

Halici Z, Borekci B, Ozdemir Y, Cadirci E, Suleyman H 2008: Protective effects of amlodipine and lacidipine on ovariectomy-induced bone loss in rats. Eur J Pharmacol 579: 241-245

Herrmann M, Seibel MJ 2008: The amino- and carboxyterminal cross-linked telopeptides of collagen type I, NTX-I and CTX-I: a comparative review. Clin Chim Acta 393: 57-75

Katagiri T, Takahashi N 2002: Regulatory mechanisms of osteoblast and osteoclast differentiation. Oral Dis 8: 147-159

Kurihara N, Ishizuka S, Kiyoki M, Haketa Y, Ikeda K, Kumegawa M 1986: Effects of 1,25-dihydroxyvitamin D3 on osteoblastic MC3T3-E1 cells. Endocrinology 118: 940-947

Lieberherr M 1987: Effects of vitamin D3 metabolites on cytosolic free calcium in confluent mouse osteoblasts. J Biol Chem 262: 13168-13173

Morain P, Peglion JL, Giesen-Crouse E 1992: Ca2+ channel inhibition in a rat osteoblast-like cell line, UMR 106, by a new dihydropyridine derivative, S11568. Eur J Pharmacol 220: 11-17

Nakatani Y, Tsunoi M, Hakeda Y, Kurihara N, Fujita K, Kumegawa M 1984: Effects of parathyroid hormone on 
cAMP production and alkaline phosphatase activity in osteoblastic clone MC3T3-E1 cells. Biochem Biophys Res Commun 123: 894-898

Nishiya Y, Sugimoto S 2001: Effects of various antihypertensive drugs on the function of osteoblast. Biol Pharm Bull 24: 628-633

Rejnmark L, Vestergaard P, Mosekilde L 2006: Treatment with beta-blockers, ACE inhibitors, and calcium-channel blockers is associated with a reduced fracture risk: a nationwide case-control study. J Hypertens 24: 581-589

Ritchie CK, Maercklein PB, Fitzpatrick LA 1994: Direct effect of calcium channel antagonists on osteoclast function: alterations in bone resorption and intracellular calcium concentrations. Endokrinology 135: 996-1003

Shimizu H, Nakagami H, Osako MK, Hanayama R, Kunugiza Y, Kizawa T, Tomita T, Yoshikawa H, Ogihara T, Morishita R 2008: Angiotensin II accelerates osteoporosis by activating osteoclasts. FASEB J 22: 2465-2475

Ushijima K, Liu Y, Maekawa T, Ishikawa E, Motosugi Y, Ando H, Tsuruoka S, Fujimura A 2010: Protective effect of amlodipine against osteoporosis in stroke-prone spontaneously hypertensive rats. Eur J Pharmacol 635: 227-230

Zahanich I, Graf EM, Heubach JF, Hempel U, Boxberger S, Ravens U 2005: Molecular and functional expression of voltage-operated calcium channels during osteogenic differentiation of human mesenchymal stem cells. J Bone Miner Res 20: 1637-164 